\title{
Serum Soluble Interleukin-2 Receptor as a Potential Biomarker for Immune-related Adverse Events
}

\author{
RYO TAKAI ${ }^{1}$, YOHEI FUNAKOSHI ${ }^{2}$, HIROTAKA SUTO ${ }^{2}$, YOSHIAKI NAGATANI ${ }^{2}$, YOSHINORI IMAMURA ${ }^{2}$, \\ MASANORI TOYODA ${ }^{2}$, KIMIKAZU YAKUSHIJIN ${ }^{2}$, NAOMI KIYOTA ${ }^{2,3}$, KEN-ICHI HARADA $^{4}$, \\ KIMIHIRO YAMASHITA ${ }^{1}$, YOSHIHIRO KAKEJI ${ }^{1}$ and HIRONOBU MINAMI ${ }^{2,3}$ \\ ${ }^{1}$ Division of Gastrointestinal Surgery, Department of Surgery, \\ Kobe University Hospital and Graduate School of Medicine, Kobe, Japan; \\ ${ }^{2}$ Division of Medical Oncology/Hematology, Department of Medicine, \\ Kobe University Hospital and Graduate School of Medicine, Kobe, Japan; \\ ${ }^{3}$ Cancer Center, Kobe University Hospital, Kobe, Japan; \\ ${ }^{4}$ Division of Urology, Department of Surgery, \\ Kobe University Hospital and Graduate School of Medicine, Kobe, Japan
}

\begin{abstract}
Background/Aim: Biomarkers for immune-related adverse events (irAEs) induced by immune checkpoint inhibitors (ICIs) are required. We encountered a patient whose skin irAE fluctuated in parallel with serum soluble interleukin2 receptor (sIL-2R). Patients and Methods: We examined 15 patients with cancer who received ICIs. Serum sIL-2R levels before and during ICI treatment were measured. The sIL-2R levels of preserved serum samples from another five patients who developed grade 3 irAEs were measured. Results: Twelve patients showed no significant changes in sIL-2R levels during $I C I$ treatment. Baseline serum sIL-2R levels in three patients increased beyond the normal range before the second cycle. These three patients had grade $\geq 2$ irAEs at the second cycle treatment visit, supporting our hypothesis. Furthermore, at diagnosis of irAEs, the sIL-2R levels of all preserved samples from patients with grade 3 irAEs were significantly elevated. Conclusion: Serum $S I L-2 R$ is a promising biomarker for the diagnosis of irAEs.
\end{abstract}

Immune checkpoint inhibitors (ICIs) that target programmed cell death-1 (PD-1) and cytotoxic T-lymphocyte antigen 4

This article is freely accessible online.

Correspondence to: Yohei Funakoshi, MD, Ph.D., Division of Medical Oncology/Hematology, Department of Medicine, Kobe University Hospital and Graduate School of Medicine, 7-5-2 Kusunoki-cho, Chuo-ku, Kobe 650-0017, Japan. Tel: +81 783825820, Fax: +81 783825821, e-mail: yohei@med.kobe-u.ac.jp

Key Words: Immune checkpoint inhibitor, immune-related adverse event, serum soluble interleukin-2 receptor.
(CTLA-4) were developed as cancer immunotherapy agents (1). Since PD-1 and CTLA-4 are inhibitory immunogenic molecules, ICIs enhance the patients' immune system to eliminate cancer cells (1). These drugs are effective in treating multiple cancers and have become staples in our arsenal of anti-cancer agents (2). However, stimulating the immune system can cause autoimmune toxicity, called immune-related adverse events (irAEs) (3).

All ICIs can induce irAEs, and the symptoms vary (3). A recent meta-analysis reported that colitis, hypophysitis, and skin rash were more frequent with anti-CTLA-4 antibodies, while pneumonitis, hypothyroidism arthralgia, and vitiligo were more common with anti-PD-1 antibodies $(4,5)$. Severe irAEs (grade $\geq 3$ ) have occurred in $28-56 \%$ and $21-32 \%$ of patients administered with anti-CTLA-4 and anti-PD-1 antibodies, respectively (4). ICIs should be discontinued in patients with persistent grade $\geq 3$ irAEs, and these cases may necessitate prolonged administration of high-dose steroids or other immunosuppressive agents to mitigate severe irAEs (3, 6-8). Thus, irAEs pose major impediments to ICI cancer immunotherapy. Although the effective management of severe irAEs is dependent upon early diagnosis and prompt intervention $(3,8)$, little is known about the risk factors for irAEs, and irAEs remain unpredictable $(3,4)$. Hence, biomarkers for early diagnosis and disease activity evaluation are required. To date, there are no available biomarkers for irAEs in daily practice.

Serum soluble interleukin-2 receptor (sIL-2R) is considered a marker of lymphocyte ( $\mathrm{T}$ and $\mathrm{B}$ cell)-mediated immune activation, and elevated levels have been recognised as a biomarker for malignant lymphoma, such as nonHodgkin's lymphoma and adult T-cell leukaemia/lymphoma (9-11). Serum sIL-2R levels are commonly measured to 
evaluate the disease state and therapeutic effect, and to detect recurrence in patients with malignant lymphoma in daily practice (9). Recently, we encountered a case in which the skin irAE activity fluctuated in parallel with changes in serum sIL-2R levels in a patient with dual cancer [follicular lymphoma (FL) and melanoma]. Based on this experience, we hypothesised that sIL-2R levels may be a potential biomarker for irAEs and undertook this research. We conducted an exploratory study to evaluate the relationship between fluctuating sIL-2R levels before and during ICI treatment and the occurrence of irAEs.

\section{Patients and Methods}

Patients and serum sIL-2R analysis. In this study, patients with malignant diseases received ICI therapy, including anti-PD-1 and antiCTLA-4 antibody combination therapy (nivolumab and ipilimumab every 3 weeks for four doses) followed by anti-PD-1 antibody monotherapy (nivolumab every 2 weeks) or anti-PD-1 antibody monotherapy (nivolumab every 2 weeks or pembrolizumab every 3 weeks), at Kobe University Hospital between May 2019 and June 2020. Each dose was decided according to the package inserts.

Serum samples were serially collected at pre-treatment, 1 week after the first cycle, and before the second cycle. Serum sIL-2R levels were measured using a chemiluminescent enzyme immunoassay (STACIA CLEIA IL-2R, LSI Medience Corp., Japan) in a fully automated immunoassay analyser (STACIA, LSI Medience Corp., Japan) at the Kobe University Hospital Clinical Laboratory. The normal range was defined as 121-613 U/ml, according to the manufacturer's instructions. We evaluated the clinical manifestations at every hospital visit. The severity of irAEs was graded using the National Cancer Institute Common Terminology Criteria for Adverse Events (version 5.0).

Ethical statements. The study design was approved by the ethics committee of Kobe University Hospital (approval number: 180152). Research was conducted in accordance with the Declaration of Helsinki and Title 45, US Code of Federal Regulations, Part 46, Protection of Human Subjects, effective December 13, 2001. All patients provided written informed consent for the use of their blood samples for research.

\section{Results}

Case report. A 67-year-old man, who received chemotherapy treatment for FL (stage IV) 9 years ago, was undergoing regular computed tomography scans and sIL-2R measurements as follow-up care for recurrence every 6 months. During the follow-up period, he was diagnosed with cutaneous melanoma with liver metastasis 1 year ago. Since lymph node swelling and sIL-2R levels were stable, we focused on treating the metastatic melanoma, and he received ICI treatment (nivolumab and ipilimumab) every 3 weeks. After two cycles of ICI treatment, skin rash was noted and a grade 3 skin irAE was diagnosed via biopsy. Notably, the sIL2R levels, which were analysed periodically for FL follow-up, were elevated after the onset of the irAE, without progression of FL. Systemic corticosteroid therapy improved the skin irAE and reduced sIL-2R levels in parallel (Figure 1). Therefore, we hypothesised that sIL-2R may be a potential biomarker for irAEs and undertook the following investigation.

Fluctuation of serum sIL-2R levels after ICI treatment. To clarify the relationship between the elevation of serum sIL$2 \mathrm{R}$ levels after ICI treatment and the occurrence of irAEs, we investigated 15 patients with cancer who received ICI treatment. Five patients were treated with nivolumab plus ipilimumab combination therapy and 10 patients were treated with anti-PD-1 antibody monotherapy. In total, three patients developed grade $\geq 2$ irAEs.

Table I shows the clinical characteristics of all 15 patients. Fluctuations in sIL-2R levels among these patients were monitored before and during ICI treatment. Twelve patients without irAEs did not show significant changes in sIL-2R levels during the treatment period. However, increased sIL$2 \mathrm{R}$ levels were observed in three patients who had grade $\geq 2$ irAEs [grade 2 thyroid dysfunction $(n=2)$ and grade 3 hepatitis $(n=1)]$ at the second cycle treatment visit, supporting our hypothesis (Figure 2).

Next, we calculated the fold-change in sIL-2R levels during the treatment period (Figure 3 ). Serum sIL-2R levels increased by 3.52-, 4.50-, and 5.82-fold in the three patients who developed irAEs, while the fold-change in sIL-2R levels ranged from 0.99 to 1.71 (median=1.20) in the other 12 patients. Moreover, in these three patients, the fold-change started to increase 1 week after the first cycle, before the diagnosis of an irAE was made.

sIL-2R levels in cryopreserved serum samples of patients with grade 3 irAEs. Cryopreserved serum samples were obtained from the five patients with grade 3 irAEs at the time of diagnosis. To validate the relationship between sIL$2 \mathrm{R}$ levels and irAEs, we measured the sIL-2R levels of these samples, all of which were found to be elevated (Table II).

\section{Discussion}

In this study, elevated sIL-2R levels were observed at the time of diagnosis of irAEs (Figures 1 and 2, Table II). These findings suggest that sIL-2R is related to irAEs. This relationship was observed regardless of the type of irAE. Moreover, the skin irAE activity changed in parallel with sIL-2R levels, as shown in Figure 1. Pathological examination of the skin showed infiltration of many T cells (data not shown). Since sIL-2R is one of the indices of immune activity, we assume that sIL-2R levels also decreased with improvement of irAE. Additionally, fold-changes in sIL-2R levels had begun to increase before irAEs developed, as shown in Figure 3. Based on these findings, we suggest that sIL-2R may be a potential biomarker for evaluating irAE activity and predicting its occurrence. 


\section{Case 1}

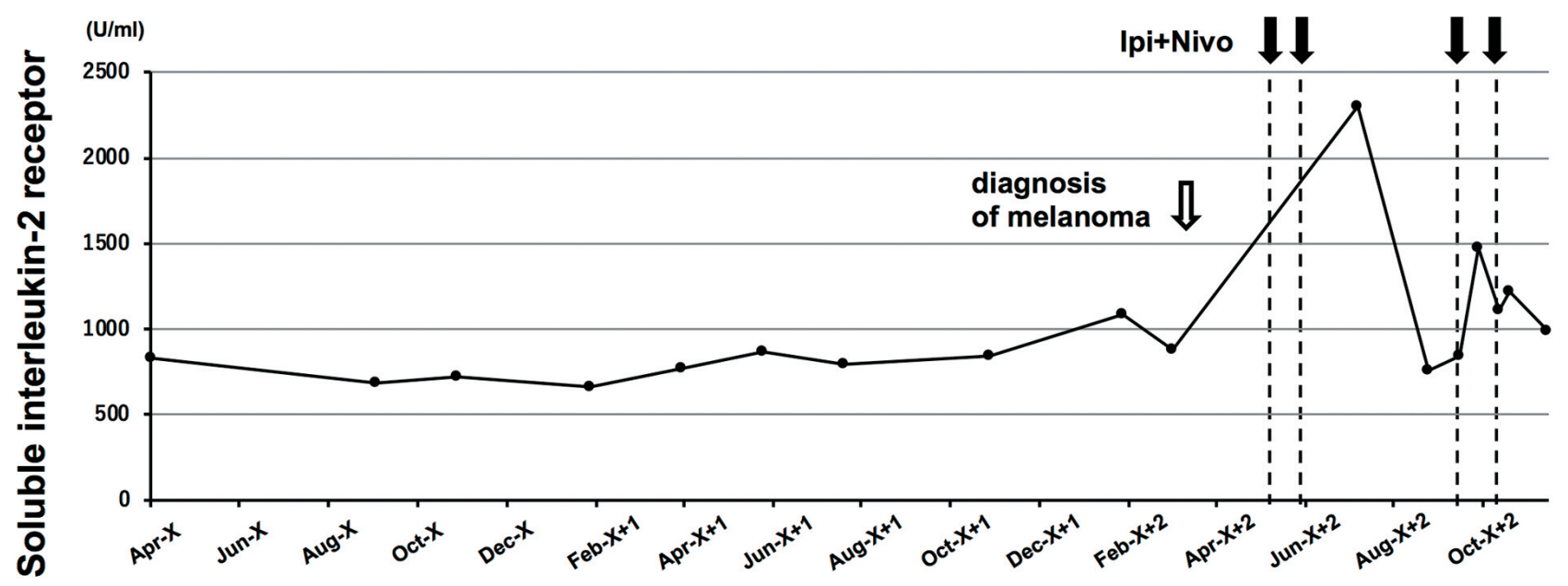

\section{Skin irAE}
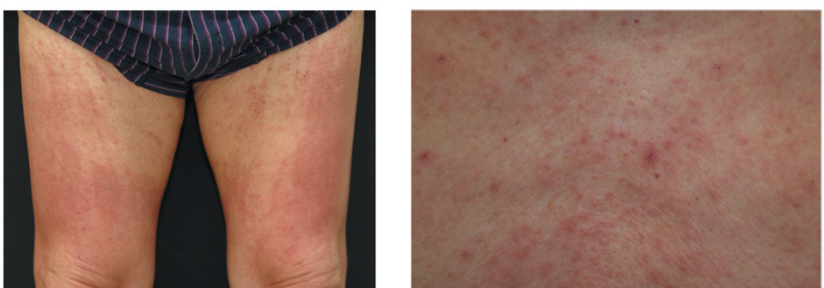

Figure 1. Clinical course. (Upper) The solid line shows the soluble interleukin-2 receptor level; the black arrows show time points of nivolumab (Nivo) and ipilimumab (Ipi) administration; the white arrow shows the time point of the diagnosis of melanoma; the black box shows systemic corticosteroid treatment; and the diagonal line shows the severity of skin immune-related adverse event (irAE). (Lower) Clinical manifestations of dermatitis at diagnosis of skin irAE.

Few studies have explored and reported candidate biomarkers for irAEs (12-14). Tanaka et al. (12) evaluated fluctuations in multiple cytokines among patients with melanoma treated with nivolumab and showed that increased serum IL-6 levels after administration were related to the development of irAEs. Tarhini et al. (13) revealed that baseline serum IL-17 levels correlated with the development of grade 3 irAEs, specifically diarrhoea/colitis, during neoadjuvant ipilimumab treatment. Because IL-17 levels are elevated in patients with inflammatory bowel disease, this finding is rational. Furthermore, Khan et al. (14) recently reported that patients who developed irAEs exhibited a greater increase in the levels of chemokines CXCL9 and CXCL10 post ICI treatment compared with patients without irAEs. Because these cytokines/chemokines are important regulators of immune activities by recruiting immune cells and promoting inflammation, they are promising biomarkers for irAEs. However, these cytokines have only been measured in cancer immunity research and not in clinical practice.
Table I. Patient characteristics.

\begin{tabular}{lccc}
\hline Characteristic & $\begin{array}{c}\text { Total } \\
(\mathrm{n}=15)\end{array}$ & $\begin{array}{c}\text { irAE Grade } \geq 2 \\
(\mathrm{n}=3)\end{array}$ & $\begin{array}{c}\text { Non-irAE } \\
(\mathrm{n}=12)\end{array}$ \\
\hline ICI & & & \\
Nivo + Ipi & 5 & 1 & 4 \\
Nivo & 7 & 2 & 5 \\
Pem & 3 & 0 & 3 \\
Age, years, & & & \\
median (range) & $(38-80)$ & $61(47-71)$ & $66.5(38-80)$ \\
Cancer type & & & 2 \\
Melanoma & 4 & 2 & 3 \\
Renal cancer & 2 & 0 & 1 \\
Head and neck cancer & 3 & 0 & 2 \\
Gastric cancer & 2 & 1 & 1 \\
Oesophageal cancer & 2 & 0 & 1 \\
Biliary tract cancer & 1 & 0 & \\
Lung cancer & 1 & &
\end{tabular}

ICI, Immune checkpoint inhibitor; Ipi, ipilimumab; irAE, immunerelated adverse event; Nivo, nivolumab; Pem, pembrolizumab. 

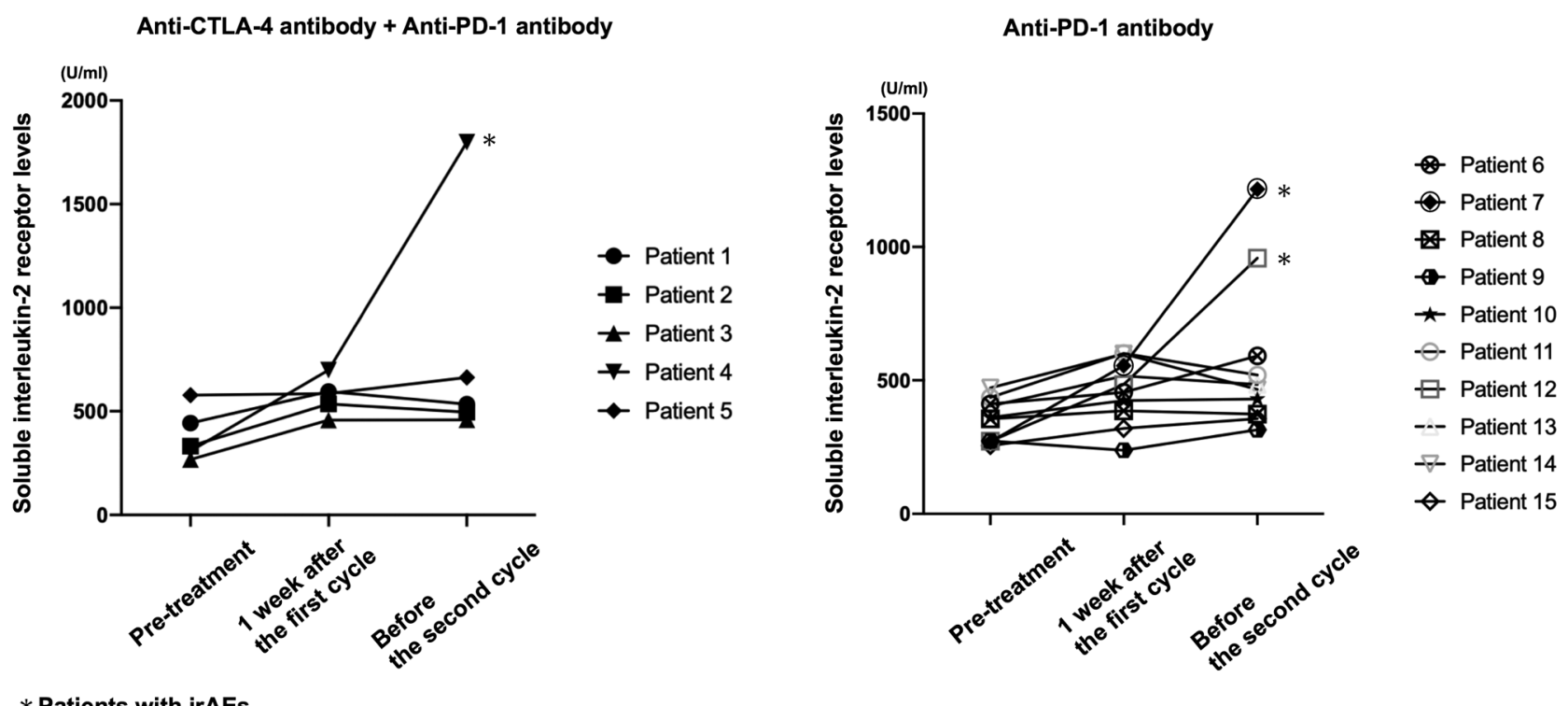

* Patients with irAEs

Figure 2. Serum soluble interleukin-2 receptor (sIL-2R) levels before and during immune checkpoint inhibitor treatment. Serum sIL-2R levels were measured at pre-treatment, 1 week after the first cycle, and before the second cycle. CTLA-4, Cytotoxic T-lymphocyte antigen 4; irAE, immunerelated adverse event; $P D-1$, programmed cell death-1.

Anti-CTLA-4 antibody + Anti-PD-1 antibody

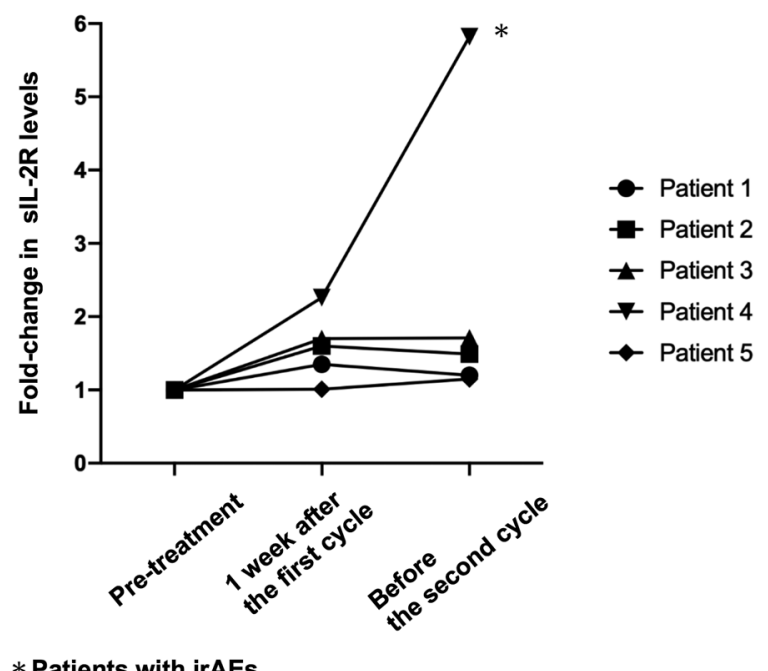

\section{Anti-PD-1 antibody}

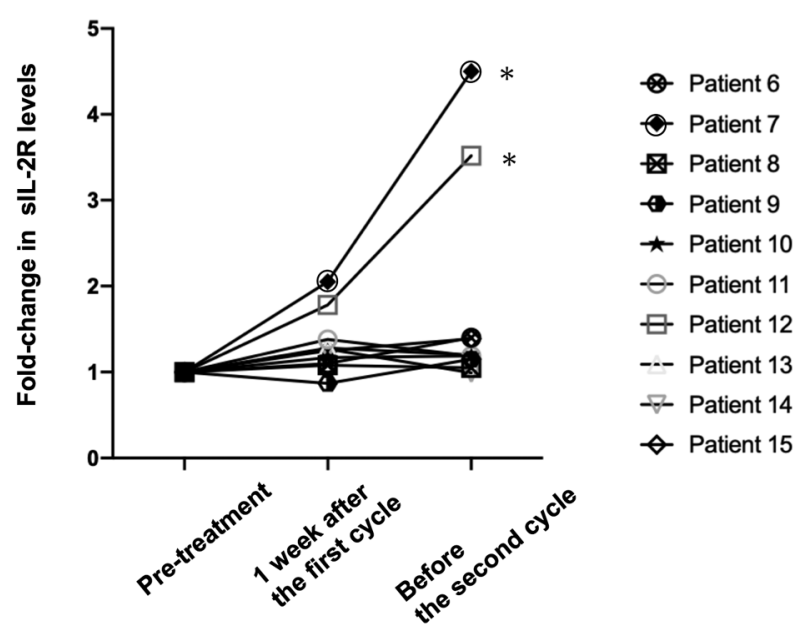

Figure 3. Fold-changes in serum soluble interleukin-2 receptor (sIL-2R) levels before and during immune checkpoint inhibitor treatment. Foldchanges were calculated at pre-treatment, 1 week after the first cycle, and before the second cycle. CTLA-4, Cytotoxic T-lymphocyte antigen 4; irAE, immune-related adverse event; PD-1, programmed cell death-1.

Serum sIL-2R levels are widely measured in daily practice in patients with malignant lymphoma (9-11), and they were reported to predict the prognosis of patients with follicular lymphoma (15). Additionally, sIL-2R levels have been correlated with various inflammatory diseases, such as viral infections, malignancies (lung and renal cancer), and autoimmune disorders (rheumatoid arthritis, systemic lupus erythematosus, sarcoidosis, and vasculitis syndrome) (16-20). In terms of the relationship between sIL-2R and irAEs, there is only one case report that correlated sIL-2R levels with the 
Takai et al: sIL-2R as a Biomarker for irAEs

Table II. Serum sIL-2R levels at the time of diagnosis of grade 3 irAEs and clinical characteristics of the other five patients.

\begin{tabular}{|c|c|c|c|c|c|}
\hline & Patient 1 & Patient 2 & Patient 3 & Patient 4 & Patient 5 \\
\hline Cancer type & Melanoma & Head and neck cancer & Melanoma & Head and neck cancer & Head and neck cancer \\
\hline ICI & Ipi following Nivo & Nivo & Ipi + Nivo & Nivo & Pem \\
\hline Age, years & 70 & 64 & 79 & 67 & 79 \\
\hline Gender & Male & Male & Male & Female & Male \\
\hline Stage & IV & IV & IV & IV & IV \\
\hline irAE $($ Grade $\geq 3$ ) & Uveitis & Hepatitis & Hepatitis & Colitis & Interstitial pneumonia \\
\hline sIL-2R* (U/ml) & 950 & 1,665 & 2,138 & 1,241 & 2,033 \\
\hline
\end{tabular}

ICI, Immune checkpoint inhibitor; Ipi, ipilimumab; irAE, immune-related adverse event; Nivo, nivolumab; Pem, pembrolizumab; sIL-2R, soluble interleukin-2 receptor. ${ }^{*}$ The normal range was defined as $121-613 \mathrm{U} / \mathrm{ml}$.

activity of irAE pneumonia (21). Our study demonstrated the relationship between sIL-2R and various types of irAEs. Because this exploratory research had a small sample size, further study is required. However, our study provides important insights into the significance of elevated sIL-2R levels at the time of diagnosis of irAEs. Moreover, measurement of sIL-2R levels may help predict the occurrence and evaluate the disease activity of irAEs. Our findings suggest that sIL-2R levels could be a potential biomarker for irAEs.

\section{Conflicts of Interest}

Hironobu Minami received research grants and honoraria from Bayer Yakuhin, Boehringer Ingelheim, Bristol-Myers Squibb, Chugai Pharmaceutical, DaiichiSankyo, Eisai, Kyowa-Kirin, Merck Serono, MSD, Novartis, Ono Pharmaceutical, Pfizer, Sanofi, Takeda Pharmaceutical, Taiho Pharmaceutical, and Eli Lilly; research grants from Asahi-Kasei Pharma, Astellas Pharma, Nippon Shinyaku, Yakult Honsha, CSL, Behring, and Nippon Kayaku; honoraria from Celgene, Ohtsuka Pharmaceutical, Shire Japan, Genomic Health, and Abbvie. Naomi Kiyota reports grants from Bristol-Myers Squibb, and Ono Pharmaceutical, during the conduct of the study; grants from Astra-Zeneca, and Roche Phamaceuticals, outside the submitted work; honoraria from Ono Pharmaceutical, Bristol-Myers Squibb, Merck Biopharma, Astra-Zeneca, Merck Sharp \& Dohme, Eisai, and Bayer. The other Authors declare no conflict of interest.

\section{Authors' Contributions}

Ryo Takai: Conception, study design, data collection, analysis, interpretation, drafting, and writing of the article; Yohei Funakoshi: conception, study design, data collection, analysis, interpretation, drafting, and writing of the article; Hirotaka Suto: data collection and interpretation; Yoshiaki Nagatani: data collection and interpretation; Yoshinori Imamura: data collection, patient management, and interpretation; Masanori Toyoda: data collection, patient management, and interpretation; Kimikazu Yakushijin: data collection, patient management, and interpretation; Naomi Kiyota: data collection, patient management, and interpretation; Ken-ichi Harada: data collection, patient management, and interpretation;
Kimihiro Yamashita: interpretation, review, and revision of the manuscript; Yoshihiro Kakeji: interpretation, review, and revision of the manuscript; Hironobu Minami: supervision of the project, interpretation, review, and revision of the manuscript. All Authors read and approved the final version of the article.

\section{References}

1 Pardoll DM: The blockade of immune checkpoints in cancer immunotherapy. Nat Rev Cancer 12(4): 252-264, 2012. PMID: 22437870. DOI: $10.1038 / \mathrm{nrc} 3239$

2 Topalian SL, Drake CG and Pardoll DM: Immune checkpoint blockade: a common denominator approach to cancer therapy. Cancer Cell 27(4): 450-461, 2015. PMID: 25858804. DOI: 10.1016/j.ccell.2015.03.001

3 Postow MA and Hellmann MD: Adverse events associated with immune checkpoint blockade. N Engl J Med 378(12): 1165, 2018. PMID: 29562154. DOI: 10.1056/NEJMc 1801663

4 Nakamura Y: Biomarkers for immune checkpoint inhibitormediated tumor response and adverse events. Front Med (Lausanne) 6: 119, 2019. PMID: 31192215. DOI: 10.3389/fmed.2019.00119

5 Khoja L, Day D, Wei-Wu Chen T, Siu LL and Hansen AR: Tumour- and class-specific patterns of immune-related adverse events of immune checkpoint inhibitors: a systematic review. Ann Oncol 28(10): 2377-2385, 2017. PMID: 28945858. DOI: 10.1093/annonc/mdx286

6 Puzanov I, Diab A, Abdallah K, Bingham CO, 3rd, Brogdon C, Dadu R, Hamad L, Kim S, Lacouture ME, LeBoeuf NR, Lenihan D, Onofrei C, Shannon V, Sharma R, Silk AW, Skondra D, Suarez-Almazor ME, Wang Y, Wiley K, Kaufman HL, Ernstoff MS and Society for Immunotherapy of Cancer Toxicity Management Working Group: Managing toxicities associated with immune checkpoint inhibitors: consensus recommendations from the Society for Immunotherapy of Cancer (SITC) Toxicity Management Working Group. J Immunother Cancer 5(1): 95, 2017. PMID: 29162153. DOI: 10.1186/s40425-017-0300-Z

7 von Itzstein MS, Khan S and Gerber DE: Investigational biomarkers for checkpoint inhibitor immune-related adverse event prediction and diagnosis. Clin Chem 66(6): 779-793, 2020. PMID: 32363387. DOI: 10.1093/clinchem/hvaa081

8 Stucci S, Palmirotta R, Passarelli A, Silvestris E, Argentiero A, Lanotte L, Acquafredda S, Todisco A and Silvestris F: Immune- 
related adverse events during anticancer immunotherapy: pathogenesis and management. Oncol Lett 14(5): 5671-5680, 2017. PMID: 29113194. DOI: 10.3892/ol.2017.6919

9 Rubin LA and Nelson DL: The soluble interleukin-2 receptor: biology, function, and clinical application. Ann Intern Med 113(8): 619-627, 1990. PMID: 2205142. DOI: 10.7326/00034819-113-8-619

10 Yasuda N, Lai PK, Ip SH, Kung PC, Hinuma Y, Matsuoka M, Hattori T, Takatsuki K and Purtilo DT: Soluble interleukin 2 receptors in sera of Japanese patients with adult $\mathrm{T}$ cell leukemia mark activity of disease. Blood 71(4): 1021-1026, 1988. PMID: 2895675

11 Wagner DK, Kiwanuka J, Edwards BK, Rubin LA, Nelson DL and Magrath IT: Soluble interleukin-2 receptor levels in patients with undifferentiated and lymphoblastic lymphomas: correlation with survival. J Clin Oncol 5(8): 1262-1274, 1987. PMID: 3114436. DOI: $10.1200 /$ JCO.1987.5.8.1262

12 Tanaka R, Okiyama N, Okune M, Ishitsuka Y, Watanabe R, Furuta J, Ohtsuka M, Otsuka A, Maruyama H, Fujisawa Y and Fujimoto M: Serum level of interleukin-6 is increased in nivolumabassociated psoriasiform dermatitis and tumor necrosis factor- $\alpha$ is a biomarker of nivolumab recativity. J Dermatol Sci 86(1): 71-73, 2017. PMID: 28069323. DOI: 10.1016/j.jdermsci.2016.12.019

13 Tarhini AA, Zahoor H, Lin Y, Malhotra U, Sander C, Butterfield LH and Kirkwood JM: Baseline circulating IL-17 predicts toxicity while TGF- $\beta 1$ and IL- 10 are prognostic of relapse in ipilimumab neoadjuvant therapy of melanoma. J Immunother Cancer 3: 39, 2015. PMID: 26380086. DOI: 10.1186/s40425015-0081-1

14 Khan S, Khan SA, Luo X, Fattah FJ, Saltarski J, GloriaMcCutchen Y, Lu R, Xie Y, Li Q, Wakeland E and Gerber DE: Immune dysregulation in cancer patients developing immunerelated adverse events. Br J Cancer Suppl 120(1): 63-68, 2019. PMID: 30377338. DOI: 10.1038/s41416-018-0155-1

15 Yamamoto M, Tanaka K, Umezawa Y, Nagao T, Toyota S and Miura O: Soluble interleukin-2 receptor level at diagnosis predicts prognosis of patients with follicular lymphoma irrespective of initial management strategy. Anticancer Res 39(9): 5115-5122, 2019. PMID: 31519623. DOI: 10.21873/anticancers.13706
16 Bien E and Balcerska A: Serum soluble interleukin 2 receptor alpha in human cancer of adults and children: a review. Biomarkers 13(1): 1-26, 2008. PMID: 17906988. DOI: $10.1080 / 13547500701674063$

17 Witkowska AM: On the role of sIL-2R measurements in rheumatoid arthritis and cancers. Mediators Inflamm 2005(3): 121-130, 2005. PMID: 16106097. DOI: 10.1155/MI.2005.121

18 Brown AE, Rieder KT and Webster HK: Prolonged elevations of soluble interleukin-2 receptors in tuberculosis. Am Rev Respir Dis 139(4): 1036-1038, 1989. PMID: 2784643. DOI: 10.1164/ajrccm/139.4.1036

19 Obara T, Vodian MA and Kung PC: Clinical significance of soluble interleukin 2 receptor for monitoring the diseases associated with activated lymphocytes and viral infections. J Clin Lab Anal 6(6): 423-436, 1992. PMID: 1432370. DOI: $10.1002 /$ jcla. 1860060615

20 Wallace DJ, Linker-Israeli M, Metzger AL and Stecher VJ: The relevance of antimalarial therapy with regard to thrombosis, hypercholesterolemia and cytokines in SLE. Lupus 2(Suppl 1): S13-S15, 1993. PMID: 8485565.

21 Yoshida K, Morishima Y, Shiozawa T, Nakazawa K, Matsuyama M, Kiwamoto T, Matsuno Y, Sekine I and Hizawa N: Serum soluble interleukin-2 receptor as a possible biomarker for the early detection and follow-up of nivolumab-induced pneumonitis. J Thorac Oncol 14(5): e90-e91, 2019. PMID: 31027748. DOI: $10.1016 /$ j.jtho.2018.12.028

Received December 13, 2020

Revised January 11, 2021

Accepted January 20, 2021 